\title{
Experimental evaluation of power control algorithms for wireless sensor networks
}

\author{
Pan Gun Park* Carlo Fischione ${ }^{* *}$ Karl H. Johansson* \\ * Automatic Control Lab, School of Electrical Engineering, Royal \\ institute of technology, Stockholm, Sweden, (e-mail: pgpark, \\ kallej@ee.kth.se) \\ ${ }^{* *}$ Department of Electrical Engineering and Computer Sciences, UC \\ Berkeley, Berkeley, CA,USA (e-mail: fischion@eecs.berkeley.edu)
}

\begin{abstract}
The main contribution of this paper is the implementation and experimental evaluation of thee radio power control algorithms for wireless sensor networks. We illustrate the necessity of lightweight radio power control algorithms for the deployment of wireless sensor networks in realistic situations. Furthermore, based on a simple loss model, we develop an algorithm that optimizes the transmit power while guaranteeing a desired packet error probability. The simple power control strategy is also compared with two other strategies in experiments using Tmote Sky sensor nodes. A component-based software implementation in the Contiki operating system is used.
\end{abstract}

\section{INTRODUCTION}

Wireless sensor networks (WSNs) are characterized by mobile nodes that can communicate with each other without a fixed infrastructure using multi-hop paths. The topology of the network may vary rapidly and unpredictably, because of fast and shadow fading. Since each node has a low battery capacity, one of the key topics is to optimize the energy consumption with respect to these variations of the network.

Energy saving techniques through routing schemes are proposed in Michail [2000] and Singli [1998]. However, these routing protocols perform radio power control using an accurate topology knowledge, and often neglect the burden of signaling overhead. In Aslam [2003], the power consumption for several wireless hardware platforms is listed. Furthermore, they suggest two complementary levels at which power consumption can be optimized: during the idle state and during the active state, when communication happens. Many ad-hoc routing protocols (e.g., AODV, DSR, DSDV, and TORA in Ya Xu [2001]) do not support power control, i.e., they assume to have fixed transmit radio power for the wireless communication. A fine tuning of the transmit power is, however, important to prolong the nodes battery life and to minimize the interference phenomena caused by neighboring nodes. In Zurita [2007], Park [2007], and Ye [2002], it was shown that the energy consumption associated radio power plays a critical role in WSNs. Radio power control should be used by each node to improve the capacity of the network by reducing the interferences among nodes.

\footnotetext{
* The work by P.G. Park and K.H. Johansson is supported by the KTH ACCESS Linnaeus Center, the Swedish Research Council, the Swedish Foundation for Strategic Research, European Commission through the Network of Excellence HYCON, and the Integrated Project SOCRADES
}

Existing hardware platforms for WSNs have limited resources in terms of memory usage and micro-controller capabilities, hence power control algorithms that require heavy computations cannot be efficiently implemented. Since each node has a single micro-controller, packets can not be received while a node is performing the processing for the power control. Most of the ad-hoc routing protocols use an acknowledgment or advertisement message in order to guarantee a required packet reception rate and to get routing table based on the path state of the network. Hence, it is possible to use these messages to carry the information related to channel estimation for radio power control.

The focus of this paper is on the performance evaluation by experiments of the radio power control algorithms, which are implemented on off-the-shelf wireless sensor nodes. We provide a description of power control as a closed-loop control system. The remainder of the paper is organized as follows: In Section 2, the system model is presented, along with the problem formulation; in Section 3, the power control algorithms are studied; in Section 4, the experimental results of the algorithms are presented for WSNs; finally, in Section 5, concluding remarks are given.

\section{SYSTEM MODEL}

Fig. 1 shows a general scenarios where nodes are connected to form a WSNs. Radio power control can be performed by pairs of transmitter-receiver nodes in the network. By minimizing the radio power consumption of the transmitter nodes of the network, the overall network consumption can be reduced. Details on the control structure are given in the following.

\subsection{Control Structure}

Fig. 2 shows the closed-loop control structure for single pair of wireless communication, i.e., one transmitter and 


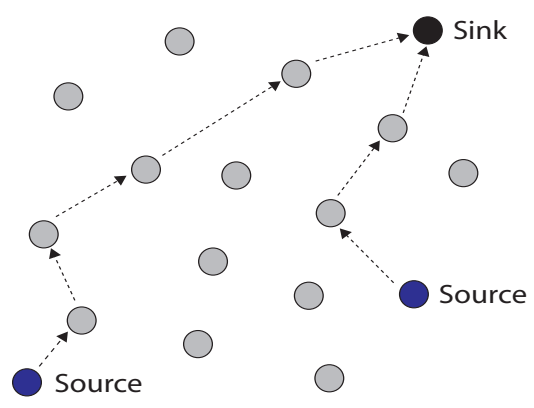

Fig. 1. Ad-hoc sensor network with source nodes transmitting over multi-hop network to a single sink.

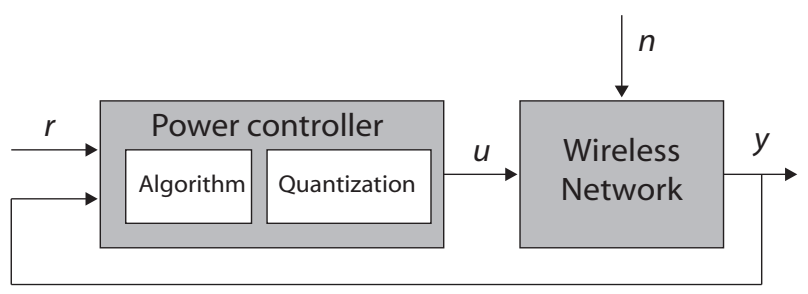

Fig. 2. A general control structure for radio power control with indication of feedback information.

one receiver node. In the power control loop, the receiver (RX) uses a quality reference $r$ associated to the signal sent by transmitter (TX). This quality reference takes into account the channel conditions. Examples of $r$ are the desired packet reception rate $\left(\mathrm{PRR}_{d}\right)$, or a receive power threshold $P_{t h r}$. The value of this statistical variable has to be estimated. According to the estimated channel condition by RX, the optimal power level is computed for the TX-RX link. Its quantized value $u$ (or PA LEVEL), depending on radio power controller, is sent to the TX by an acknowledgement message or other signalling packets commonly used in routing and MAC protocols. Furthermore, if we assume the wireless channel is reciprocal in the link between TX and RX, the signalling packet from the RX to the TX can be transmitted using the same power level. Such a signalling packet reaches the TX after a small delay, which accounts for processing operations and communication. After TX receives the signalling packet, it retrieves the optimal power level and actuates the transmit power level toward the RX. In Fig. 2, we denoted with $n$ the noise at the receiver.

\subsection{Problem Formulation}

The problem we are targeting is finding suitable power control algorithms for the control structure in Fig. 2. The algorithms should meet two requirements in addition to providing a low power consumption for the wireless transmission: (i) minimizing the overhead used by power control; (ii) be implementable through a component-based software design. Specifically, the power control problem consists in regulating the radio power at the transmitters such that a given performance of the communication $y$ is experienced at the receiver. Examples of $y$ are the Received Signal Strength Indicator (RSSI) of the Tmote sensor nodes, Tmote [2006], or the packet reception rate (PRR).

\section{THREE POWER CONTROL ALGORITHMS}

In this section, we discuss three power control algorithms, Multiplicative-Increase Additive-Decrease Power Control (MIAD PC), Packet Error Rate Power Control (PER PC) and Simple Channel Model Power Control (SCM PC). The parameters of the MIAD PC can be fixed or adaptive. The MIAD and PER PC were proposed in Zurita [2007]. The signals of the power control algorithms are described in Tab. 1. In the following subsections, the algorithms are described in detail.

Table 1. Control Signals of the three power control algorithms.

\begin{tabular}{|c||c|c|c|}
\hline Signal & $r$ & $u$ & $y$ \\
\hline \hline MIAD PC & PRR $_{d}$ & PA LEVEL & PRR \\
\hline Adaptive MIAD PC & $P_{t h r}, \mathrm{PRR}_{d}$ & PA LEVEL & PRR, RSSI \\
\hline PER PC & PRR $_{d}$ & PA LEVEL & RSSI \\
\hline SCM PC & $P_{t h r}, \mathrm{PRR}_{d}$ & PA LEVEL & RSSI \\
\hline
\end{tabular}

\subsection{MIAD Power Control}

The MIAD PC is based on the following mechanism to set the transmission power level $u(t)$. When an erroneous packet is detected, i.e. satisfying the quality reference $r$ $\left(\mathrm{PRR}_{d}\right)$, the power $P_{i}$ of node $i$ is increased by $\alpha(t) \Delta$, where $\alpha(t)$ is an integer and $\Delta$ the step size. Each correctly received packet imposes a decrease of the transmit power by $\beta(t) \Delta$. The parameters $\alpha(t), \beta(t)$ and $\Delta$ obviously influence performance of the packet error rate process, and the power consumption. In the MIAD PC, these parameters are fixed but in the adaptive MIAD PC, the parameters $\alpha(t)$ and $\beta(t)$ vary as follows

$$
\begin{aligned}
& \alpha(t)=\frac{R_{\mathrm{in}} y(t)}{P_{t h r}}, \\
& \beta(t)=\frac{R_{\mathrm{de}} P_{t h r}}{y(t)},
\end{aligned}
$$

where $R_{\text {in }}$ and $R_{\text {de }}$ are constant value, $y(t)$ is the signal strength of the received packet at time $t$ and $P_{t h r}$ is the sensitivity of radio controller. Note that the time $t$ indicates the event of receiving the packet. The adaptive MIAD PC adapts $\alpha(t)$ and $\beta(t)$ to the received signal strength $y(t)$. Therefore, adaptive MIAD PC may converge to the optimal power level faster than MIAD PC.

The implementation of the adaptive MIAD PC requires knowledge of the packet error rate, once a power level is fixed. If the packets are numbered, the receiver can estimate the packet error probability, and then the optimal power levels as computed by the adaptive MIAD PC can be sent by a control packet. The solution is simple and requires light computation by the micro-controller of each node. However, a disadvantage of this solution is that a node needs to observe the channel during some time to accurately estimate the packet error rate. Furthermore, since it requires a certain amount of transmitted packets to make the estimation, there is a trade-off between observation time and traffic load. In Section 4, we present an implementation of the adaptive MIAD PC with a realtime estimation of the packet error rate. 


\subsection{PER Power Control}

The PER PC is based on an analytical model of the wireless channel to estimate the Signal to Interference plus Noise Ratio (SINR) for each communication link. By setting a constraint on $r\left(\mathrm{PRR}_{d}\right)$, the optimal transmit power $u(t)$ can be derived for each wireless link considering the channel condition. Furthermore, the packet error probability can be computed according to the modulation scheme and the wireless propagation model. Since we have used the sensor node Tmote Sky in the experiments reported later on in this paper, the PER of O-QPSK (offset quadrature phase shift keying) modulation is considered. After each node receives a packet, it derives the SINR based on the $y(t)$ (RSSI), which is a link performance metric provided by the Tmote Sky. In this way, the PER PC adapts the channel condition faster than MIAD PC, where, on the contrary, a node has to wait a fixed period to count the number of received packet. The drawback of the PER PC is that the estimation of SINR based on $y(t)$ (RSSI) may be difficult on computationally constrained sensor nodes, since some accurate signal processing is required to estimate the average and standard deviation of the transmitted and interfering signals, see Zurita [2007] for details.

\subsection{SCM Power Control}

The SCM PC is based on the simple Additive white Gaussian noise (AWGN) model of the wireless channel to derive the transmit power instead of estimating the SINR of the received signal. The channel attenuations can be derived using the $y(t)$ (RSSI) of the received signal and the transmit power level $u(t)$. From the channel attenuation and the threshold sensitivity $P_{t h r}$ of the receiver node $r$, it is possible to derive the value of the transmit power $u(t)$. Specifically, let us denote the received signal power at time $t$ with $y(t)$, the transmit radio power with $u(t)$ and channel attenuation with $P_{\text {loss }}(t)$. Then, the received power $y(t)$ is given by

$$
y(t)=u(t)+P_{\text {loss }}(t) .
$$

The received power can be successfully detected only if $y(t) \geq r$. If the previous condition is not satisfied, the received signal is in outage. Hence, the transmit power to use, such that no outage is experienced, is given by $u(t)=r-P_{\text {loss }}(t)$. The question is how to estimate the channel attenuation term $P_{\text {loss }}(t)$. In static indoor environments the channel attenuation at time $t$ can be well approximated by the attenuation at the previous time instance $P_{\text {loss }}(t-1)$. In the experimental results section, we provide evidence for this approximation. Therefore, a simple algorithm can be derived to find a suitable transmit power $u(t)$ for each link:

$$
u(t)=r-P_{\text {loss }}(t)+\epsilon
$$

where the $\epsilon$ is the offset considering fading wireless condition and the $P_{\text {loss }}(t)$ is approximated as follows

$$
P_{\text {loss }}(t) \approx y(t-1)-u(t-1) .
$$

Note that $\epsilon=\sigma Q^{-1}\left(1-P_{s}\right)$, where $\sigma$ is the standard deviation of shadow fading, $\mathrm{Q}$ function and $P_{s}$ denotes the required packet receiving rate. After the node receives

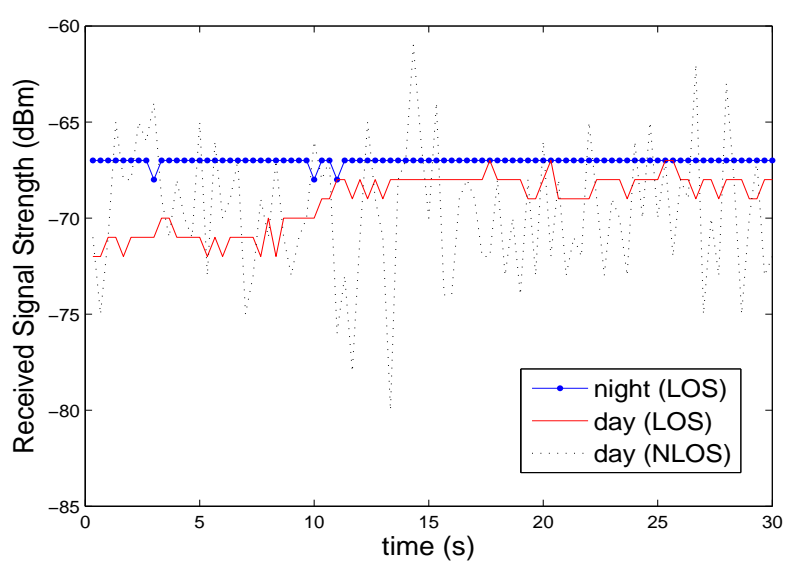

Fig. 3. Variation of the RSSI over time for an indoor environment during day and night time, and with nodes within Line of Sight (LOS) and Non Line of Sight (NLOS).

a packet, it retrieves the power level and evaluates the channel loss and power level $u(t)$. The node sends the derived optimal transmit power to the transmitter using a signalling packet.

\section{EXPERIMENTAL RESULTS}

This section presents the main contribution of this paper, which is an experimental evaluation of three power control algorithms. The algorithms are implemented on Contiki OS using Tmote Sky. The Contiki OS allows for threadbased programs running on top of an event-based kernel with dynamic loading and unloading of individual components, which results in a flexible software architecture suitable for resource-constrained systems. We first compare the algorithms for a fixed position of the transmitter and receiver within Line of Sight (LOS) and Non Line of Sight (NLOS), and then for different distances within LOS. Finally, we estimate the total energy consumption of a node for each algorithm.

In order to evaluate the performance of the power control algorithms, we compared them with the case of fixed maximum power level $(0 \mathrm{dBm})$, i.e., the case of no radio power control. The signaling packets sent by the sink node to the source node are beacons with a periodicity of one beacon every $1 \mathrm{~s}$ in MIAD PC. We set $R_{\text {in }}=0.002$ and $R_{\mathrm{de}}=7$ in Equation (1) and Equation (2) for the MIAD $\mathrm{PC}$. The sink node can detect packet losses by knowing the source packet generation rate. The source node generates packets with a fixed traffic rate of 10 pckts/s. Since the expected number of packets to be received is more than $9 \mathrm{pcks} / \mathrm{s}$, the difference between this value and the received number of packets gives the estimation of the packet error rate.

The experiments are conducted in an indoor office environment with wireless LAN communication interfering with the WSN. For each experiment, the source node is located at $4.5 \mathrm{~m}$ from the sink node. We considered static and timevarying conditions of the wireless channel. The static case corresponds to a fixed position of the sink, which is located in LOS with the source. In this case, the wireless channel 


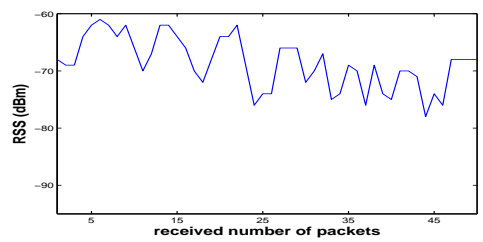

(a) RSSI in MIAD PC.

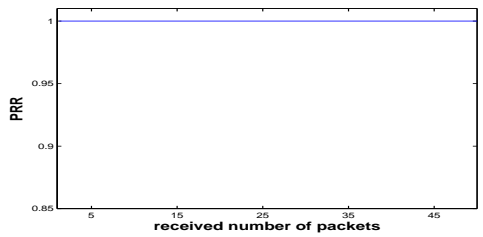

(d) PRR in MIAD PC.

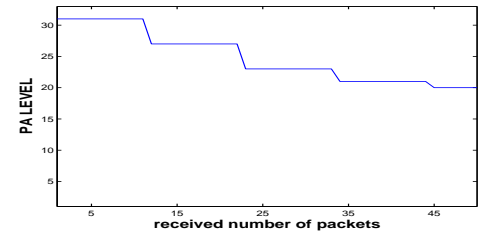

(g) Power level in MIAD PC.

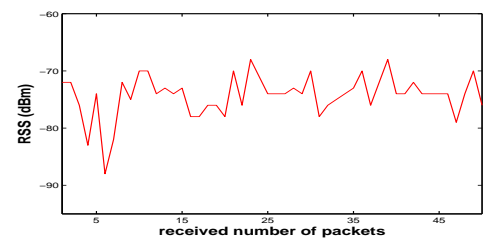

(b) RSSI in PER PC.

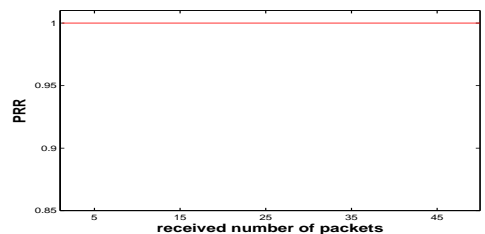

(e) PRR in PER PC.

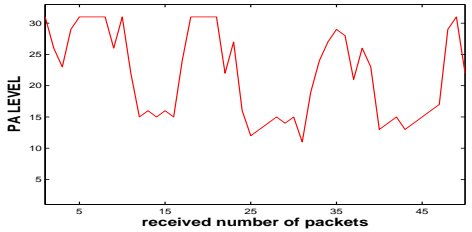

(h) Power level in PER PC.

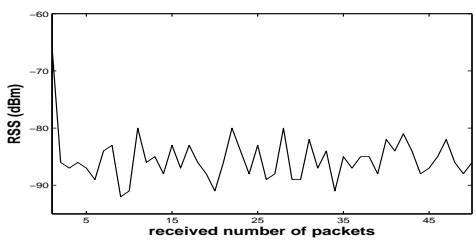

(c) RSSI in SCM PC.

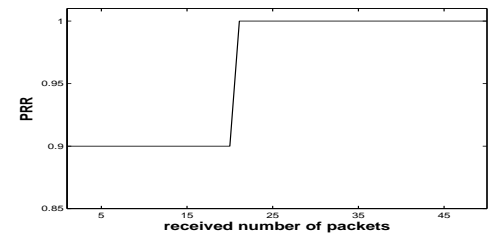

(f) PRR in SCM PC.

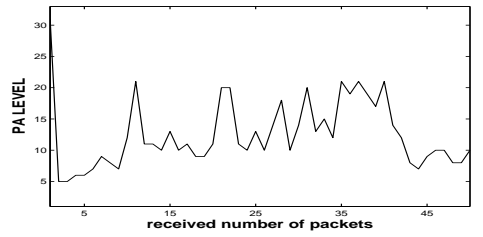

(i) Power level in SCM PC.

Fig. 4. Experimental results (RSSI, power level, PRR) for the three power control algorithms studied in this paper as function of received number of packets in a Rayleigh fading environment. The RSS is used for signal $y$ for all three algorithms and MIAD PC uses both PRR and RSS. The radio power level refers to the signal $u$ for all three algorithms.

is well described by an AWGN model. In the time-varying case, the sink node is let to move around its initial position, along a line of $10 \mathrm{~cm}$, with a speed of $10 \mathrm{~cm}$ per second. Furthermore, a metal object is put in front of the sink, so the source and the sink are not in line-of-sight. The motion of the sink on a short distance allows us to neglect the variation of the wireless channel due to shadow fading and path loss, so the channel can be well described by a Rayleigh fading distribution. In Fig. 3, the RSSI measured for each case of the wireless propagation is reported. In the figure, the LOS lines corresponds to an AWGN channel, while the NLOS line corresponds to a Rayleigh channel. From the figure, it is possible to conclude that the channel coefficients are slowly time varying for the AWGN case, so that SCM PC can be applied.

Figs. 4 report the RSSI, transmit power level, and PRR for the power control algorithms in Rayleigh fading environment. Specifically, we plotted the evolutions of RSSI, power level, and PRR for a number of 50 packets. MIAD $\mathrm{PC}$ is reported in Fig. 4(a), (g), and (d). PER PC is plotted in Fig. 4(b), (h) and (e). SCM PC is reported in Fig. 4(c), (i), and (f). The RSSI, the plots (a), (d) and $(\mathrm{g})$, is different for each power control case becouse the algorithms were run in different moments. As a first general remark, it can be observed that the power levels have quite a different behavior over time for the power control algorithms. In particular, Fig. 4(g) shows that the decreasing of power level for the MIAD PC goes very slow, even though the PRR is very good (see Fig. 4(d)). Hence, the power levels in MIAD PC converge slower than PER and SCM PC. This is due to the high variability of the RSSI, caused by the Rayleigh fading. The high variance of the RSSI causes slow convergence of MIAD parameter in Equation (1) and Equation (2).

It is possible to compute a correlation coefficient between the power levels and the RSSI. From the experimental data of PER PC, a negative correlation can be inferred, given by -0.6412 . The curve of the transmit power level in Fig. 4(i) also presents negative correlation with RSSI curve in Fig. 4(c), where the correlation coefficient is -0.4629 . It is interesting to observe that RSSI values of SCM PC is oscillating around $-90 \mathrm{dBm}$ in Fig. 4(c), whereas RSSI curve of PER PC is mostly higher than $-80 \mathrm{dBm}$ in Fig. 4(c). This is due to the minimum RSSI imposed by SCM PC, and recalling that the Tmote Sky have a sensitivity of $-95 \mathrm{dBm}$.

To characterize quantitatively the power consumption, we define the gain from using power control for node $i$ as

$$
\rho_{i}=\frac{P_{\max }}{P_{\mathrm{avg}, i}}
$$

where $P_{\max }=0 \mathrm{dBm}$ is the radio output power corresponding to the maximum power level in Tmote Sky, and $P_{\text {avg, } i}$ is the average power consumed by node $i$ during the experiment. A high $\rho_{i}$ and a low packet error rate indicate a good behavior of the power control algorithm.

In Tab. 2, we report the energy gain and the PRR for power control algorithms in both the cases of AWGN environment and Rayleigh environment. In the table, we compare the performance of the PC algorithms with the case of maximum transmit power (MAX). As first remark, it is interesting to observe that the Rayleigh fading requires more radio power with respect to the AWGN 
Table 2. Gain and packet error rate for two different propagation conditions.

\begin{tabular}{|c||c|c|c|c|}
\hline \multicolumn{1}{|c||}{} & \multicolumn{2}{c|}{ AWGN } & \multicolumn{2}{c|}{ Rayleigh } \\
\hline & $\rho_{i}$ & PRR $\%$ & $\rho_{i}$ & PRR \% \\
\hline \hline MAX & 1 & 100 & 1 & 100 \\
\hline MIAD PC & 1.6662 & 97.1 & 1.5247 & 88.5 \\
\hline PER PC & 1.8114 & 99.6 & 1.2206 & 100 \\
\hline SCM PC & 1.9974 & 99.7 & 1.5368 & 95.8 \\
\hline
\end{tabular}

case. This is due to the fact that in Rayleigh propagation environments, deep attenuations of the wireless channel coefficients must be compensated with larger transmit powers. The gain of power control increases for SCM PC when it is compared to PER and MIAD PC, whereas the packet reception rate is over $97 \%$ for all algorithms. On the contrary, considering MIAD and SCM PC in Rayleigh fading channel, the MIAD PC has higher packet error rate and, at the same time, higher energy consumption. The PER PC has higher energy consumption than the others, but at the same time, highest PRR $100 \%$. Hence, there is an intrinsic tradeoff between the gain of power control and packet error rate.

In Fig. 5, the experimental results obtained for one pair of nodes using different power control algorithms as function of distance in AWGN environment are reported. In this set of experiments, a source was placed at $2 \mathrm{~m}$ intervals along a straight line away from a sink. The source sent packets at the rate of 10 packets per second. This process was repeated for transmitters located from $1 \mathrm{~m}$ to $15 \mathrm{~m}$ from the sink. After the sink collected 600 packets at each distance, we compared the performance of three algorithms in terms of power gain and PRR. Looking Fig. 5, it is interesting to observe that MIAD PC has lower PRR with respect to the PER and SCM PC and, at the same time, lower power gain. This is due to the fact that in MAID PC, the packet loss is compensated after estimating the PRR in certain period. Therefore, the adaptability of MIAD PC is slower than two algorithms. The PER PC has similar PRR as SCM PC, whereas the power gain is lower than SCM PC. This can be explained considering that the SINR of PER PC is obtained through an estimation which provides a lower value with respect to the true one. Note that lower SINR assigns higher transmit power, and, vice versa, high radio powers give high SINR. On the contrary, SCM PC has higher packet reception rates and, at the same time, higher power gain through the whole measuring range except at the distance of $7 \mathrm{~m}$. This is due to the fact that at the distance $7 \mathrm{~m}$, deep attenuations and higher fluctuations of wireless channel are experienced, because of metal door and glass window in the corridor. It is interesting to observe that assigning the maximum power level does not guarantee the highest packet reception rate in deep fading wireless channels.

To compare the performance of the power control algorithms in terms of overall energy consumption, we estimated the cumulative energy consumption of the sensor nodes. In our investigation, we assume a first order circuit model to derive the energy consumption for the different modes of operation of the sensor nodes, Heinzelman [2000]. The energy consumption $E_{\text {tot }}(t)$ to transmit and receive a packet between a pair of nodes at the time $t$ is estimated as

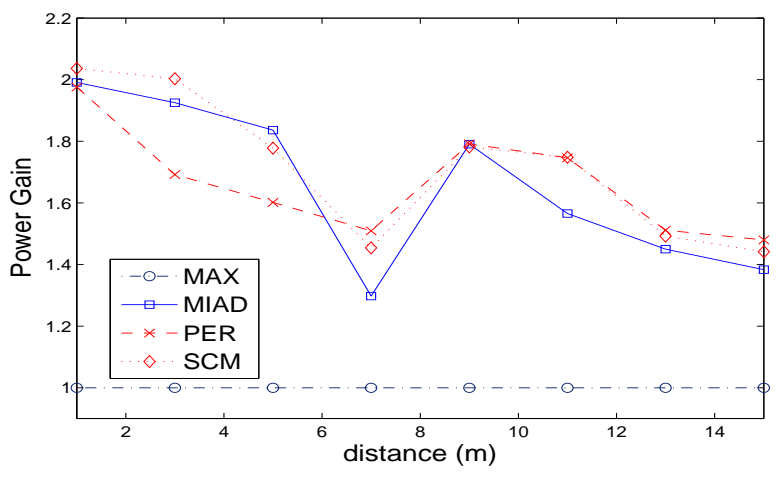

(a) Power gain of the power control algorithms.

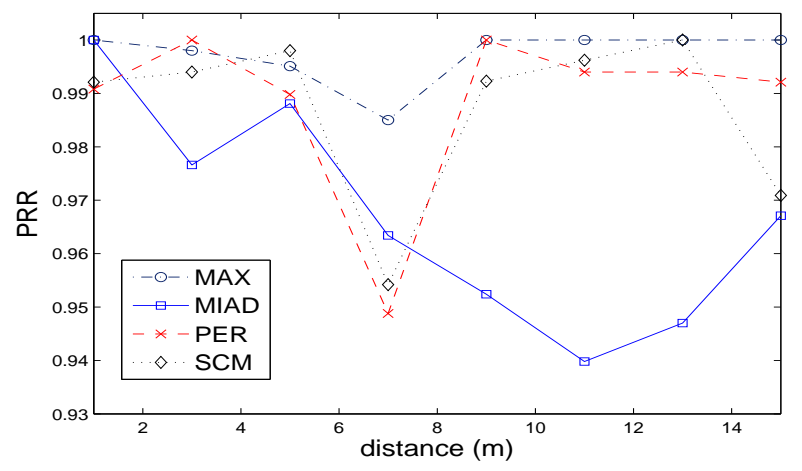

(b) Packet Reception Rate (PRR) of the power control algorithms.

Fig. 5. Performance of three power control algorithms at different distances in AWGN environment.

$$
E_{\mathrm{tot}}(t)=E_{T X} l+P_{\mathrm{con}}(t) \frac{l}{R}+E_{R X} l,
$$

where $R$ is the transmission rate, $P_{\text {con }}(t)$ is the power consumption depending on transmit power level and packet length $l, E_{T X}$ is the energy spent to run the transmitter circuitry, and $E_{R X}$ is the energy spent to run the receiver circuitry. The first two terms in (7) reflect the energy consumption to transmit a $l$-bit packet using the simple circuit model, and last term describes the consumption to receive the packet. Values for the Tmote Sky are $R=250 \mathrm{kbps}$, $E_{T X}=234.0 \mathrm{~nJ} / \mathrm{bit}, E_{R X}=261.6 \mathrm{~nJ} /$ bit, Tmote [2006]. Note that, with these value of the parameters, receiving a message is not a low cost operation.

To measure the energy consumption of different power control algorithms, we estimate the cumulative energy consumption as follows

$E_{\text {cum }}(t+1)= \begin{cases}E_{\text {cum }}(t)+E_{\text {tot }}(t+1) & \text { if success } \\ E_{\text {cum }}(t)+E_{T X} l+P_{\text {con }}(t) \frac{l}{R} & \text { otherwise }\end{cases}$

where the condition "if success" denotes the successful packet reception. We assume that communication error in feedback control system comes from a failed data transmission. In the derivation of the measure of the energy consumption, note that the signalling packet sent from the destination to the source has the same size of data packets.

Fig. 6(a) shows the cumulative energy consumption for the three radio power control algorithms, and the case of 


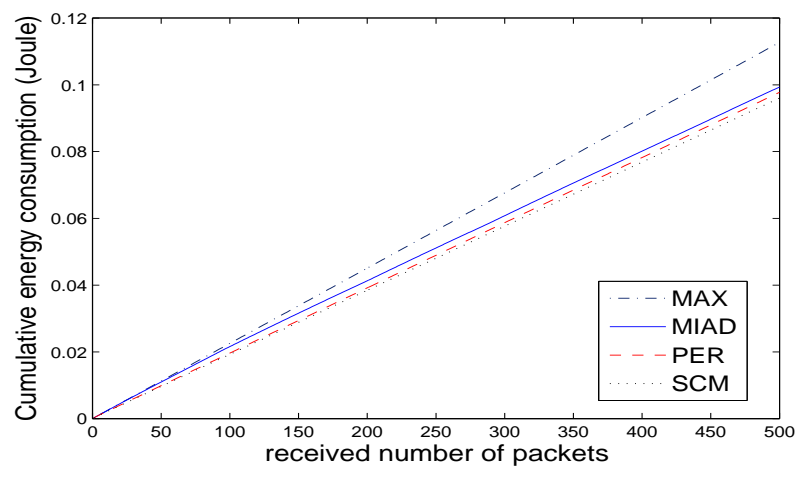

(a) AWGN environment.

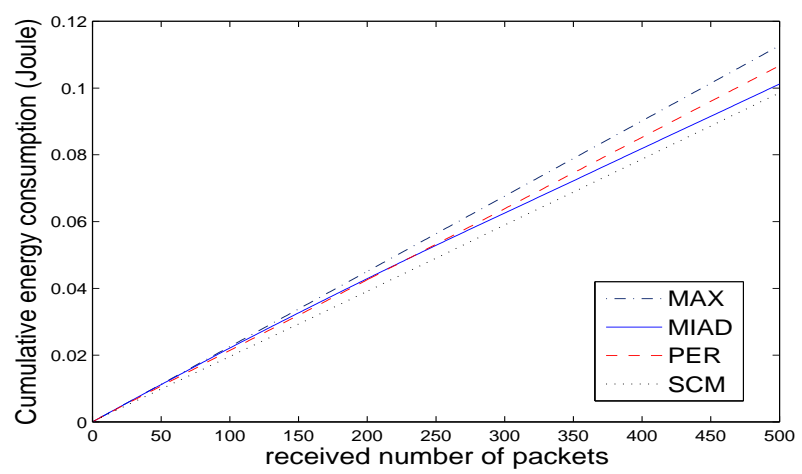

(b) Rayleigh fading environment.

Fig. 6. Cumulative Energy Consumption for the three power control algorithms as function of received number of packets in different environments.

transmission with the maximum power level. Note that the cumulative energy consumption is dependent on the traffic rate and distance between source and sink. The MIAD, PER and SCM PC consume similar amount of energy due to the stable wireless channel in AWGN environment. Observing Tab. 2, the cumulative energy consumption matches the power gain of AWGN environment. However, under Rayleigh fading conditions, the SCM PC is more energy efficient than MIAD and PER PC, as shown in Fig. 6(b). Observe that the energy consumption of MIAD and PER PC are similar during the initial packet transmission. Recalling Fig. 4, this is due to the slow adaptability of MIAD PC, and at the same time the PER $\mathrm{PC}$ assigns large transmit powers. Note that the Rayleigh fading conditions requires more power with respect to the AWGN case.

\section{CONCLUSIONS}

Simple feedback control systems for radio power control in WSNs have been implemented and evaluated in this paper. Specifically, we have reviewed two power control mechanisms: one based on a MIAD mechanism, and another one based on the packet loss probability. Furthermore, we have studied a simple power control algorithm which is based on AWGN model of the wireless channel. We have compared the performance of the three algorithms in terms of packet error rate, power gain, and energy efficiency in two different environments, AWGN and Rayleigh environment. The experimental results show that SCM PC have good performance in terms of energy consumption and packet error rate than PER and MIAD PC in AWGN environment. Furthermore, even though SCM PC outperforms the energy efficiency in both AWGN and Rayleigh environment, PER PC may be recommended in Rayleigh environment because of stable PRR than SCM PC. Ongoing work is focused on the integration of the power control algorithms to existing routing protocol, so that knowledge of the wireless propagation scenario can be beneficial to the reduction of the power consumption in the routing of packets.

\section{REFERENCES}

B. Zurita Ares, P. G. Park, C. Fischione, A. Speranzon and K. H. Johansson. On Power Control for Wireless Sensor Networks: System Model, Middleware Component and Experimental Evaluation. European Control Conference, Greece, 2007.

J. Aslam, Q. Li, D. Rus. Three power-aware routing algorithms for sensor networks. Wireless Communications and Mobile Computing, Volume 2, Number 3, Pages 187-208, March 2003.

Ya Xu, J. S. Heidemann, D. Estrin. Geography-informed Energy Conservation for Ad Hoc Routing. Mobile Computing and Networking, Pages 70-84, 2001.

N. Patwari, and A. O. Hero III. Using Proximity and Quantized RSS for Sensor Localization in Wireless Networks. Proceedings of the 2nd ACM international Conference on Wireless Sensor Networks and Applications, September 2003.

Tmote Sky Data Sheet, Moteiv, San Francisco, CA,2006. [Online]Available:http://www.moteiv.com/products/d ocs/tmote-sky-datasheet.pdf.

A. Dunkels, B. Grönvall, T. Voigt. Contiki - a lightweight and fexible operating system for tiny networked sensors. In Proceedings of the First IEEE Workshop on Embedded Networked Sensors, 2004.

P. G. Park. Protocol Design of Sensor Networks for Wireless Automation, Master Thesis, Royal Institute of Technology (KTH), Stockholm, Sweden, January 2007.

W. Ye, J. Heidemann, D. Estrin. An energy-efficient MAC Protocol for Wireless Sensor Networks. In Proceesding of IEEE INFOCOM 2002.

A. Michail, A. Ephrernides. Energy-efficient Routing for Connection-Oriented Traffic in Ad-Hoc Wireless Networks. In Procedings of PIMRCOO, Vol 1.2, Pages 762766, London, March 2000.

S. Singli, M. Woo, C.S. Raghavendra. Power-aware Routing in Mobile Ad Hoc Networks. Mobile Computing and Networking, Pages 181-190, 1998.

W. Heinzelman, A. Chandrakasan, and H. Balakrishnan, Energy-efficient Communication Protocol for Wireless Microsensor Networks. System Sciences, Vol. 2, Pag. 10, 2000.

T. S. Rappaport. Wireless Communications: Principles and Practice. Prentice-Hall, 2nd ed., December 2001.

C. Bettstetter and C. Hartmann. Connectivity of Wireless Multihop Networks in a Shadow Fading Environment. ACM International Workshop on Modeling, Analysis and Simulation of Wireless and Mobile Systems (MSWiM), September 2003. 